\title{
Cytokine Networks in Ulcerative Colitis
}

\author{
Giulia Roda, Margherita Marocchi, Alessandro Sartini, and Enrico Roda \\ Department of Clinical Medicine, Gastroenterology Unit, S. Orsola-Malpighi Hospital, Via Massarenti 9, 40138 Bologna, Italy
}

Correspondence should be addressed to Giulia Roda, giuliaroda@gmail.com

Received 15 September 2010; Accepted 27 December 2010

Academic Editor: Hiromi Sanada

Copyright () 2011 Giulia Roda et al. This is an open access article distributed under the Creative Commons Attribution License, which permits unrestricted use, distribution, and reproduction in any medium, provided the original work is properly cited.

\begin{abstract}
Ulcerative colitis (UC) is a relapsing inflammatory bowel disease whose pathogenesis has yet to be defined completely. A genetic predisposition is needed to develop the colitis, but environmental factors are necessary to trigger an exaggerated and aberrant immune response, which stands at the basis of the mucosal healing. Cytokines, small cell-signaling protein molecules secreted by various types of cells including immune and glia cells, are the main mediators of the mucosal healing in IBD; ulcerative colitis is characterized by a Th2 atypical immune response, since, beside the classical proinflammatory cytokines, such as IL-1, IL-6, and TNF- $\alpha$, in the pathogenesis of UC, we find a complex network in which the Th2 cytokines, IL-10 and IL-13, play a key role, but little IL-4 was found. Our aim was to review the literature to point out the state of the art in terms of cytokines because the knowledge of cytokine network in UC could lead to the discovery of new therapeutical targets.
\end{abstract}

\section{Introduction}

Ulcerative colitis (UC), a form of disease belonging to the socalled inflammatory bowel diseases (IBDs), is characterized by continuous inflammation of the intestinal lamina propria, starting from the rectum and potentially involving the whole colonic mucosa.

The course of UC is typically unpredictable; it is a chronic disease characterized by spontaneous remittances and relapses [1].

At present, its pathogenesis is still unclear, but evidence suggests that the disease occurs in genetically susceptible subjects, and it is trigged by environmental factors, which lead to an exaggerated and uncontrolled immune response [2-9].

However, genetic susceptibility itself is not sufficient to explain the development of IBD; in fact, environmental triggers have been identified as agents able to disrupt or at least to affect the mucosal barrier, such as NSAIDs, antibiotics, and viral and bacterial infections [10]. Actually, disease expression seems to be the consequence of a dysregulated immune response to both bacteria and bacterial products in a susceptible host; in fact, while inflammatory response against luminal antigens is suppressed in healthy individuals, a destructive immune response is initiated in IBD patients.
In this context, the immune response plays a key role in the initiation, augmentation, and perpetuation of the disease. In IBD, in fact, there is a loss of immune tolerance towards the luminal antigens and the commensal flora.

This aberrant immune response is mediated by different cytokines, small cell-signaling protein molecules secreted by various types of cells including immune and other cells such as glia cells, which stimulate the proliferation of antigen-specific effector cells in order to activate the adaptive immune system, with consequent burst of local and systemic inflammation.

Cytokines play many different roles in IBD, such as production of inflammatory mediators, nitric oxide, leukotrienes, platelet-activating factor, activation of NF$\kappa \mathrm{B}$ pathway, and inhibition of apoptosis; thus, cytokines themselves are directly responsible for mucosal injury and consequent tissue damage. However, some of them induce a disease-specific immune response in UC and CD; so, we can identify UC-associated specific cytokine networks [11].

\section{UC Is Associated with a Th2-Atypical Response}

Ulcerative colitis shows a particular immune pathway; in the past, it was thought to be a Th2 dominant disease, but in 
the last years, many studies proved that UC is characterized by a Th2 atypical immune response. In fact, no IL- 4 was detected in UC tissues, but both IL-13 and IFN- $\gamma$ were found at high levels [12].

The CD4+ lymphocytes had been firstly characterized in Th1 and Th2 cells, according to their function and to the production of specific cytokines; recently, an additional Th subset, Th17, has been described. The Th1 cells are activated by IL- 12 secreted by the antigen-presenting cells and produce classical proinflammatory cytokines (IFN- $\gamma$ and TNF- $\alpha$ ) and IL-2.

Thus, the Th1-mediated immune response is mainly directed towards intracellular pathogens as it is meant to secrete factors that promote intracellular killing by macrophages (superoxide, nitric oxid) or that activate cytotoxic lymphocytes [10].

The Th2 cells produce IL-4, IL-5, IL-6, IL-10, and IL13 and promote atopy through activation of mast cells and induction of IgE responses.

The Th1 and Th2 subpopulations are mutually regulated; IFN- $\gamma$ can in fact downregulate (drop down) the Th2mediated responses, while IL-4, IL-10, and IL-13 inhibit the Th1-mediated responses.

\section{Different Cytokines Are Involved in UC Pathogenesis}

Beside the classical proinflammatory cytokines, such as IL-1, IL- 6 , and TNF- $\alpha$, in the pathogenesis of UC we find a complex network in which the Th2 cytokines, IL-10 and IL-13, play a key role.

3.1. TNF- $\alpha$. TNF- $\alpha$ is a typical proinflammatory cytokine produced by APCs and macrophages; its role in the promotion of inflammation is performed by the production of IL- $1 \beta$ and IL- 6 , the expression of adhesion molecules, the proliferation of fibroblasts, the activation of procoagulant factors, the cytotoxicity of the acute phase response, and inhibition of apoptosis $[13,14]$.

Moreover, TNF- $\alpha$ activates neutrophils and macrophages, stimulates B cells, and increases the production of IFN- $\gamma$ by $\mathrm{T}$ cells [15].

The increased expression of TNF- $\alpha$ has been demonstrated by several studies in intestinal biopsies, both in CD patients and in those with UC versus healthy controls; in addition, mucosal biopsies from affected areas showed significantly higher levels than those of mucosal biopsies from macroscopically unaffected areas. These data therefore demonstrate a direct correlation between TNF- $\alpha$ and inflammation.

Finally, serum levels of TNF- $\alpha$ correlate with clinical and laboratory indices of disease activity such as the erythrosedimentation rate (ESR), C-reactive protein (CRP), and disease activity index (DAI) [16].

3.2. IL-4 and TGF- $\beta$. IL-4 and TGF $\beta$ are anti-inflammatory cytokines and their role in IBD is yet to be characterized.
IL-4 is a stimulatory molecule for both B and T cells, and it is produced mainly by activated lymphocytes. Its main role stands in the inhibition of the formation of colonies of macrophages, of the production of $\mathrm{H}_{2} \mathrm{O}_{2}$ by monocytes, and of the release of mediators such as IL- $1 \beta$ and TNF$\alpha$.

The levels of IL-4 and IL-4 mRNA were found reduced in IBD, demonstrating the loss of balance between anti and proinflammatory cytokines in favor of the proinflammatory ones [17].

The role of IL-4 in the reduction of inflammation was also demonstrated by Griga et al., whose studies showed that the administration of IL- 4 causes a significant reduction of VEGF produced by peripheral blood mononuclear cells in patients with active $\mathrm{CD}$ and UC, with a plain antiinflammatory effect [18].

TGF- $\beta$ is an inhibitory cytokine, a key regulator in the maintenance of immune and inflammatory responses.

Evidence suggests that TGF- $\beta$ acts together with growth factors in protecting host tissue from luminal changes and can help the mucosal healing in IBD $[19,20]$.

However, CD and UC show distinct patterns of production of TGF- $\beta$; patients with UC show an increased synthesis of TGF- $\beta 1$ by mononuclear cells of the lamina propria, compared to $\mathrm{CD}$ patients and healthy controls, emphasizing that although TGF- $\beta$ acts as an anti-inflammatory molecule in the systemic immune system, locally it shows proinflammatory properties [21].

3.3. IL-6. IL-6 is a typical proinflammatory cytokine whose production is induced during the acute-phase response; it is secreted by a large number of cells, and its main sources in the gut are the macrophages [22].

IL-6 exerts most of its proinflammatory effects by binding to its soluble receptor (sIL-6R), and this binding stimulates only cells that express gp130 but not IL-6R; this process is called transsignalling, and it occurs through the STAT3 molecule (signal transducer and activator of transcription-3).

Increased IL-6 levels were found in both biopsy samples and sera of UC and CD patients [23].

3.4. IL-10. IL-10 is an anti-inflammatory cytokine produced by T cells, $\mathrm{B}$ cells, and monocytes; in presence of an antigenic stimulus, IL-10 inhibits the production of IL- $1 \beta$, IL-6, and TNF- $\alpha$ [24] by diminishing the antigen-presenting capacity of monocytes via downregulation of MHCII [25].

The protective role played by IL-10 within the mucosal immune system is confirmed by data showing that inactivation of the gene for IL-10 in mice causes a chronic ileocolitis with increased production of IL-12 and IFN- $\gamma[26,27]$.

The analysis of cytokine mRNA levels by real-time quantitative reverse transcription-polymerase chain reaction (RT-PCR) in T lymphocytes of UC patients and healthy controls showed a significant increase of IL-10 in UC, while it was undetectable in healthy colonic lamina propria $[28,29]$.

A study by Melgar et al. showed a highly significant increase of IL-10 mRNA levels in T lymphocytes and 
an increased frequency of IL-10-positive cells in UC; IL-10 mRNA levels were also elevated in $\mathrm{T}$ lymphocytes of the noninflamed ileum and correlated with disease activity at both locations.

A recent study by Szkaradkiewicz et al. compared the levels of circulating inflammatory cytokines and of IL-10 of IBD patients to the ones of healthy controls, represented by both disease-free patients and patients affected by colorectal cancer (CRC) [30].

UC patients showed high levels of proinflammatory cytokines, but the concentration of IL-10 $(4,40 \pm 1,55)$ was significant as well, compared to healthy subjects $(1,35 \pm$ $0,96)$. Between $\mathrm{CD}$ and UC, differences were found only in TNF- $\alpha$ (higher in CD group) and IL-10 (higher in UC group) values. Moreover, the UC group showed lower levels of TNF- $\alpha$ and lower levels of IL-10 compared to patients affected by colorectal cancer, while cytokine concentrations found in $\mathrm{CD}$ group did not show any significant difference. These data confirm that both IBD and colorectal cancer induce an increase in inflammatory cytokines production; IL-10 concentration does not show any variation in $\mathrm{CD}$ and CRC group compared to healthy controls, while it is increased in UC group. However, this IL-10 concentration increase is not enough to inhibit properly the secretory activity of monocytes and macrophages.

3.5. IL-13. Lamina propria lymphocytes of UC patients show increased levels of IL-5 and IL-13, restricted to the inflamed areas; these data confirm the crucial role of the Th2 immune response in the pathogenesis of UC.

Recently, it was shown that IL-13 is produced by Cd-1 reactive natural killer $\mathrm{T}$ cells and that IL-13 acts on the epithelial cells and make them disfunctional.

IL-13 is a typical Th2 cytokine which is responsible for epithelial barrier damage, speed of mucosal repair, and alteration of the tight junctions, with a consequent impairment of the mucosal permeability.

Particularly, Heller et al. performed a series of studies in order to determine exactly the effects of IL-13 in UC. They showed that IL-13 does not induce cell necrosis, but it contributes to cell apoptosis, with a significant increase of the conductance in the apoptotic areas [31].

Moreover, the impairment of cell permeability was investigated; the addition of IL-13 to cell cultures increases the flux of ions and of large molecules such as mannitol and lactulose. The composition of tight junctions was altered as well; the WB analysis performed on colonic biopsy specimens from UC patients, and controls showed a 10-fold increase in claudin-2, while the expression of occludin, claudin-1, and claudin-4 was lower than in controls.

IL-13 also affects the speed of mucosal repair; under physiological conditions, gaps in the mucosal layer can be closed by neighboring cells, and the addition of IL-13 to cells colures where mucosal lesions were artificially induced reduces the speed of this restitution of a $30 \%$.

\section{Cytokine Networks: A Potential Therapeutical Target}

Given the role of cytokines in the pathogenesis and in the perpetuation of inflammatory bowel diseases, the use of cytokines and anticytokines shows great promise in the treatment of this kind of disease.

The biological treatment with Infliximab, a chimeric monoclonal antibody against TNF- $\alpha$, was firstly proposed for the treatment of $\mathrm{CD}$, but it was shown to be effective in patients with steroid-refractory UC as well, where it was expected to be not functional as the mucosal levels of TNF- $\alpha$ are not increased in UC $[32,33]$.

Infliximab efficacy in UC seems to be due to an apoptotic effect on T cells and on APCs which secrete cytokines. Since lately the role of IL-13 in the pathogenesis of UC was emphasized, it is now thought that the effectiveness of antiTNF- $\alpha$ is related to its apoptotic effect on cells which express TNF- $\alpha$ on their surface, and these cells could be the ones that secrete proinflammatory cytokines responsible for the mucosal damage, such as IL-13.

Moreover, the humanized, non-Fc-binding immunoglobulin G2 anti-CD3 monoclonal antibody Visilizumab is now under evaluation as a treatment for UC steroidrefractory patients [1].

Yamamoto et al. proposed the use of a monoclonal antibody against IL-6R in an experimental induced colitis murine model, and their data showed a significant dicrease in IFN- $\gamma$, TNF- $\alpha$, and IL- $1 \beta$ mRNA. The remittance of this induced colitis seems to be due to the inhibition of the circulating leucocytes and to the increase of the T-cells apoptotic rate, both induced by the antibody itself [34].

Moreover, the IL-6/STAT3 signalling pathway, required for the activation of the mucosal T cells, might be one of the forthcoming therapeutical targets [35].

\section{Conclusion}

Ulcerative colitis is a chronic inflammatory bowel disease characterized by continuous inflammation of the intestinal lamina propria, starting from the rectum and potentially involving the whole colonic mucosa. Its pathogenesis involves many different factors, such as genetical susceptibility, environmental triggers, and immune response, which are all necessary, but none of them is sufficient itself to induce the disease.

Cytokines surely play a key role in the initiation, augmentation, and perpetuation of the disease, since they are directly responsible for the mucosal injury.

We reviewed the state of art concerning the main cytokine profiles involved in UC pathogenesys; UC is characterized by a Th2 atypical immune response, with high levels of IL-6, IL-10, and IL-13, beside the classical proinflammatory cytokines.

The understanding of these cytokine networks may lead to important developments both in the diagnostical and therapeutical phase. Firstly, a deeper knowledge of these networks is required to better understand the pathogenesis 
of UC; then, from a diagnostical point of view, the analysis of cytokine expression profiles could let the identification of new markers which could work as predictors of disease onset, disease exacerbation, or disease phenotype.

Finally, a more specific evaluation of cytokines involved in UC tissue damage could lead to the development of targeted and tailored therapies.

\section{Conflict of Interests}

The authors declare no conflict of interests.

\section{References}

[1] S. R. Targan and L. C. Karp, "Defects in mucosal immunity leading to ulcerative colitis," Immunological Reviews, vol. 206, pp. 296-305, 2005.

[2] D. K. Podolsky, "Inflammatory bowel disease," The New England Journal of Medicine, vol. 325, pp. 928-937, 1991.

[3] S. Targan and F. Shanahan, Inflammatory Bowel Disease: From Bench to Bedside, Williams \& Wilkins, Baltimore, Md, USA, 1st edition, 1994.

[4] M. Parkes, M. M. Barmada, J. Satsangi, D. E. Weeks, D. P. Jewell, and R. H. Duerr, "The IBD2 locus shows linkage heterogeneity between ulcerative colitis and Crohn disease," American Journal of Human Genetics, vol. 67, no. 6, pp. 16051610, 2000.

[5] H. Toyoda, S. J. Wang, H. Y. Yang et al., "Distinct associations of HLA class II genes with inflammatory bowel disease," Gastroenterology, vol. 104, no. 3, pp. 741-748, 1993.

[6] J. Hampe, S. Schreiber, S. H. Shaw et al., "A genomewide analysis provides evidence for novel linkages in inflammatory bowel disease in a large European cohort," American Journal of Human Genetics, vol. 64, no. 3, pp. 808-816, 1999.

[7] T. Ahmad, J. Satsangi, D. Mcgovern, M. Bunce, and D. P. Jewell, "Review article: the genetics of inflammatory bowel disease," Alimentary Pharmacology and Therapeutics, vol. 15, no. 6, pp. 731-748, 2001.

[8] M. Roussomoustakaki, J. Satsangi, K. Welsh et al., "Genetic markers may predict disease behavior in patients with ulcerative colitis," Gastroenterology, vol. 112, no. 6, pp. 1845-1853, 1997.

[9] J. Satsangi, K. I. Welsh, M. Bunce et al., "Contribution of genes of the major histocompatibility complex to susceptibility and disease phenotype in inflammatory bowel disease," Lancet, vol. 347, no. 9010, pp. 1212-1217, 1996.

[10] L. Mayer, "Evolving paradigms in the pathogenesis of IBD," Journal of Gastroenterology, vol. 45, no. 1, pp. 9-16, 2010.

[11] F. Sanchez-Muñoz, A. Dominguez-Lopez, and J. K. Yamamoto-Furusho, "Role of cytokines in inflammatory bowel disease," World Journal of Gastroenterology, vol. 14, no. 27, pp. 4280-4288, 2008.

[12] G. Bouma and W. Strober, "The immunological and genetic basis of inflammatory bowel disease," Nature Reviews Immunology, vol. 3, no. 7, pp. 521-533, 2003.

[13] H. Baumann and J. Gauldie, "The acute phase response," Immunology Today, vol. 15, no. 2, pp. 74-80, 1994.

[14] B. Begue, H. Wajant, J. C. Bambou et al., "Implication of TNFrelated apoptosis-inducing ligand in inflammatory intestinal epithelial lesions," Gastroenterology, vol. 130, no. 7, pp. 1962 1974, 2006.
[15] M. Bosani, S. Ardizzone, and G. B. Porro, "Biologic targeting in the treatment of inflammatory bowel diseases," Biologics, vol. 3, pp. 77-97, 2009.

[16] J. M. Reimund, C. Wittersheim, S. Dumont et al., "Mucosal inflammatory cytokine production by intestinal biopsies in patients with ulcerative colitis and Crohn's disease," Journal of Clinical Immunology, vol. 16, no. 3, pp. 144-150, 1996.

[17] A. Andoh, Y. Yagi, M. Shioya, A. Nishida, T. Tsujikawa, and Y. Fujiyama, "Mucosal cytokine network in inflammatory bowel disease," World Journal of Gastroenterology, vol. 14, no. 33, pp. 5154-5161, 2008.

[18] T. Griga, U. Hebler, E. Voigt, A. Tromm, and B. May, "Interleukin-4 inhibits the increased production of vascular endothelial growth factor by peripheral blood mononuclear cells in patients with inflammatory bowel disease," HepatoGastroenterology, vol. 47, no. 36, pp. 1604-1607, 2000.

[19] S. Kanazawa, T. Tsunoda, E. Onuma, T. Majima, M. Kagiyama, and K. Kikuchi, "VEGF, basic-FGF, and TGF- $\beta$ in Crohn's disease and ulcerative colitis: a novel mechanism of chronic intestinal inflammation," American Journal of Gastroenterology, vol. 96, no. 3, pp. 822-828, 2001.

[20] I. C. Lawrance, L. Maxwell, and W. Doe, "Inflammation location, but not type, determines the increase in TGF- $\beta 1$ and IGF-1 expression and collagen deposition in IBD intestine," Inflammatory Bowel Diseases, vol. 7, no. 1, pp. 16-26, 2001.

[21] B. Del Zotto, G. Mumolo, A. M. Pronio, C. Montesani, R. Tersigni, and M. Boirivant, "TGF- $\beta 1$ production in inflammatory bowel disease: differing production patterns in Crohn's disease and ulcerative colitis," Clinical and Experimental Immunology, vol. 134, no. 1, pp. 120-126, 2003.

[22] G. Rogler and T. Andus, "Cytokines in inflammatory bowel disease," World Journal of Surgery, vol. 22, no. 4, pp. 382-389, 1998.

[23] J. M. Reimund, C. Wittersheim, S. Dumont et al., "Mucosal inflammatory cytokine production by intestinal biopsies in patients with ulcerative colitis and Crohn's disease," Journal of Clinical Immunology, vol. 16, no. 3, pp. 144-150, 1996.

[24] M. Bosani, S. Ardizzone, and G. B. Porro, "Biologic targeting in the treatment of inflammatory bowel diseases," Biologics, vol. 3, pp. 77-97, 2009.

[25] G. Rogler and T. Andus, "Cytokines in inflammatory bowel disease," World Journal of Surgery, vol. 22, no. 4, pp. 382-389, 1998.

[26] R. Kuhn, J. Lohler, D. Rennick, K. Rajewsky, and W. Muller, "Interleukin-10-deficient mice develop chronic enterocolitis," Cell, vol. 75, no. 2, pp. 263-274, 1993.

[27] D. M. Rennick and M. M. Fort, "Lessons from genetically engineered animal models. XII. IL-10-deficient (IL-10(-/-) mice and intestinal inflammation," American Journal of Physiology, vol. 278, no. 6, pp. G829-G833, 2000.

[28] S. Schreiber, T. Heinig, H. G. Thiele, and A. Raedler, "Immunoregulatory role of interleukin 10 in patients with inflammatory bowel disease," Gastroenterology, vol. 108, no. 5, pp. 1434-1444, 1995.

[29] S. Melgar, M. M. W. Yeung, A. Bas et al., "Over-expression of interleukin 10 in mucosal $\mathrm{T}$ cells of patients with active ulcerative colitis," Clinical and Experimental Immunology, vol. 134, no. 1, pp. 127-137, 2003.

[30] A. Szkaradkiewicz, R. Marciniak, I. Chudzicka-Strugała et al., "Proinflammatory cytokines and IL-10 in inflammatory bowel disease and colorectal cancer patients," Archivum Immunologiae et Therapiae Experimentalis, vol. 57, no. 4, pp. 291-294, 2009. 
[31] F. Heller, P. Florian, C. Bojarski et al., "Interleukin-13 is the key effector Th2 cytokine in ulcerative colitis that affects epithelial tight junctions, apoptosis, and cell restitution," Gastroenterology, vol. 129, no. 2, pp. 550-564, 2005.

[32] B. E. Sands, W. J. Tremaine, W. J. Sandborn et al., "Infliximab in the treatment of severe, steroid-refractory ulcerative colitis: a pilot study," Inflammatory Bowel Diseases, vol. 7, no. 2, pp. 83-88, 2001.

[33] T. Ochsenkühn, M. Sackmann, and B. Göke, "Infliximab for acute, not steroid-refractory ulcerative colitis: a randomized pilot study," European Journal of Gastroenterology and Hepatology, vol. 16, no. 11, pp. 1167-1171, 2004.

[34] M. Yamamoto, K. Yoshizaki, T. Kishimoto, and H. Ito, "IL-6 is required for the development of Th1 cell-mediated murine colitis," Journal of Immunology, vol. 164, no. 9, pp. 4878-4882, 2000.

[35] R. Carey, I. Jurickova, E. Ballard et al., "Activation of an IL-6:STAT3-dependent transcriptome in pediatric-onset inflammatory bowel disease," Inflammatory Bowel Diseases, vol. 14, no. 4, pp. 446-457, 2008. 


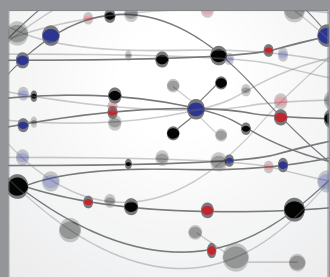

The Scientific World Journal
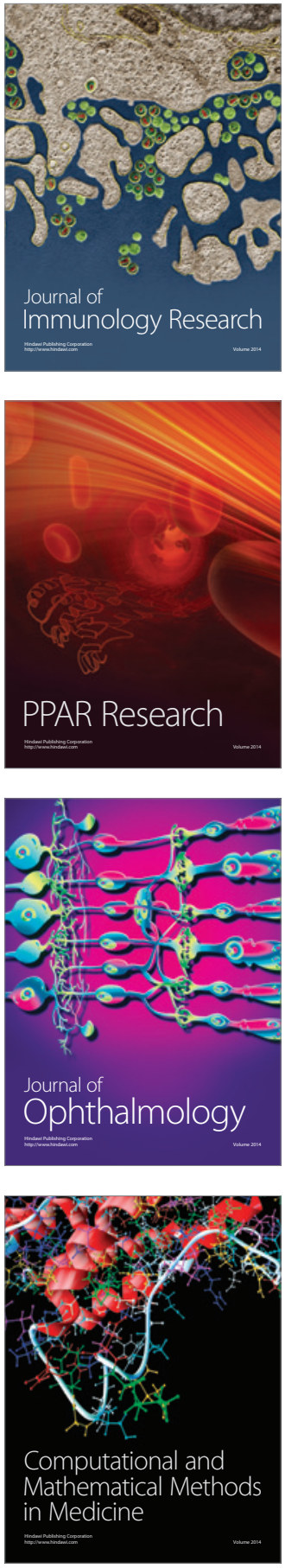

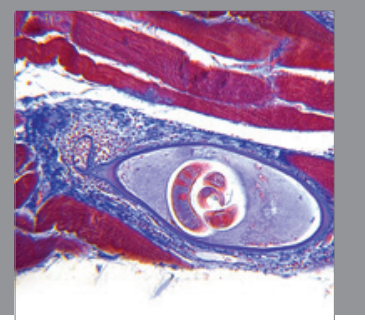

Gastroenterology

Research and Practice
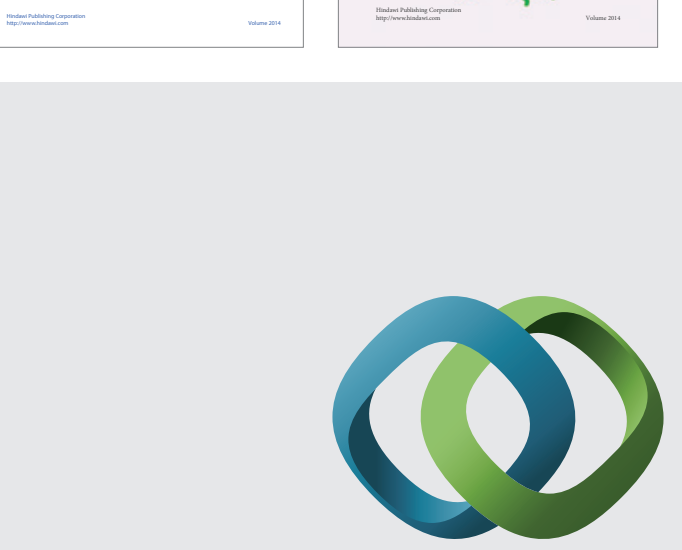

\section{Hindawi}

Submit your manuscripts at

http://www.hindawi.com
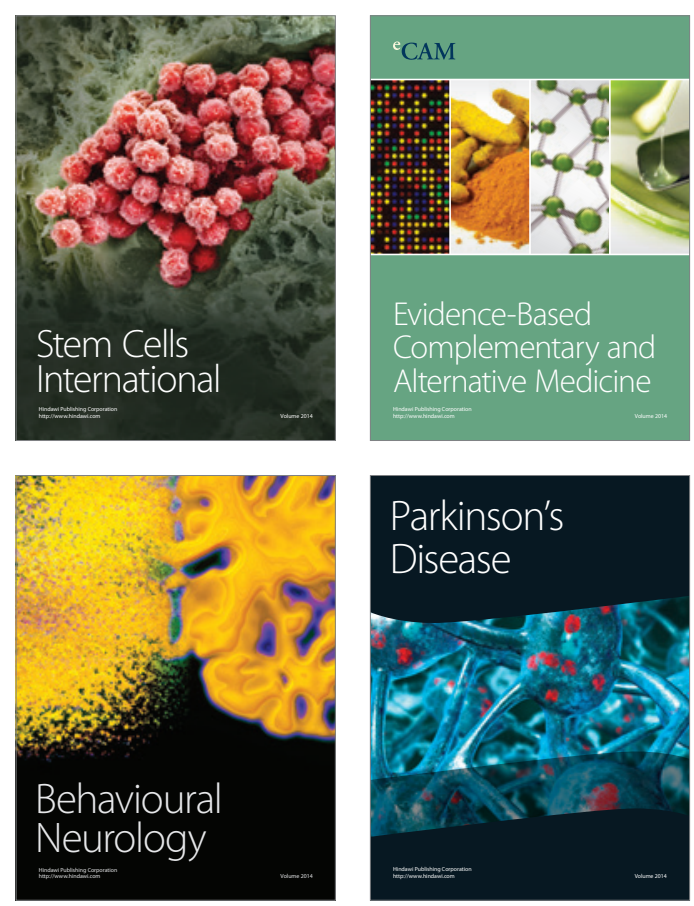

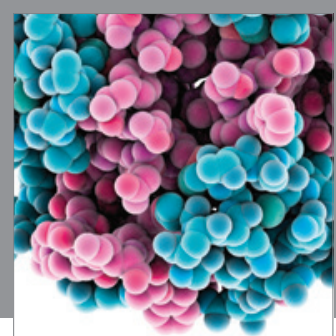

Journal of
Diabetes Research

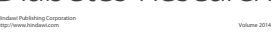

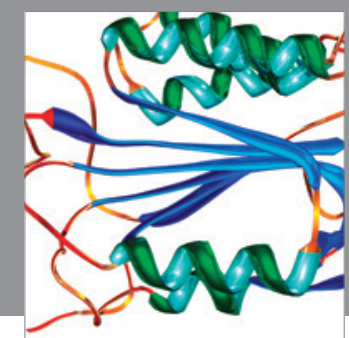

Disease Markers
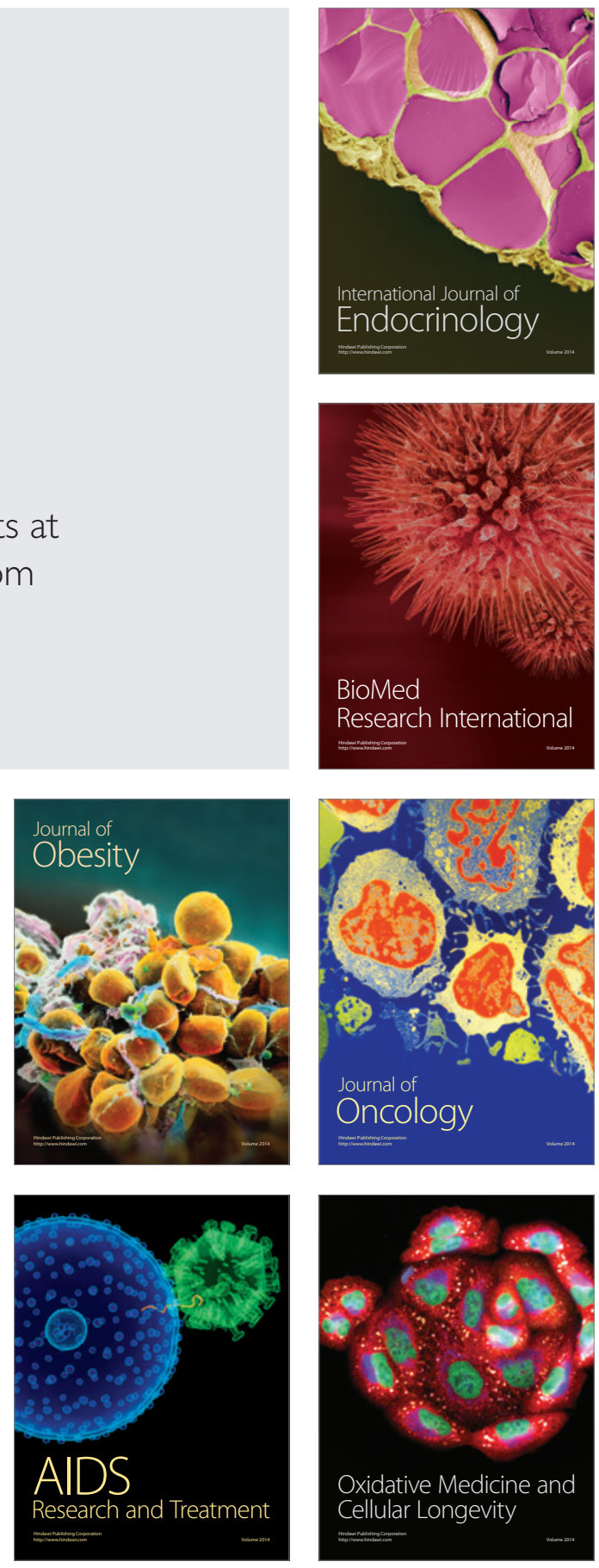